\title{
Elements of an Automatic Data Scientist
}

\author{
Luc De Raedt, Hendrik Blockeel, Samuel Kolb, Stefano Teso, and Gust \\ Verbruggen \\ Department of Computer Science, KU Leuven, Belgium \\ firstname.lastname@cs.kuleuven. be
}

\begin{abstract}
A simple but non-trivial setting for automating data science is introduced. Given are a set of worksheets in a spreadsheet and the goal is to automatically complete some values. We also outline elements of the SYNTH framework that tackles this task: SYNTH-A-SIZER, an automated data wrangling system for automatically transforming the problem into attribute-value format; TACLE, an inductive constraint learning system for inducing formula's in spreadsheets; MERCs, a versatile predictive learning system; as well as the autocompletion component that integrates these systems.
\end{abstract}

Keywords: Automated data science $\cdot$ Autocompletion $\cdot$ Data wrangling · Learning constraints · Versatile models.

\section{Introduction}

The field of artificial intelligence (AI) can be viewed as the endeavor to automate all tasks that require intelligence when performed by humans $[16,18]$. As scientific activities do require intelligence, artificial intelligence researchers [14] have been developing robot scientists. While robot scientists typically target the natural sciences, this paper focuses on the automation of data science. Given the abundance of data, the needs to analyse data and the challenges in hiring data scientists, even partial automation of data science would make a radical impact on business. Indeed, automated data analysts are viewed as potentially the second most impactful AI-powered technology by business executives [1].

Automated data science is not really new as there have been many approaches to automating different aspects of data science, machine learning and data mining. However, these approaches are typically embedded in existing tools and workbenches that offer a multitude of operations, learning algorithms and parameter settings as well as graphical user interfaces visualizing particular workflows [20]. When the user specifies the task of interest (e.g., predicting a particular field), the intelligent data analysis assistant will then suggest a particular workflow (or sequence of algorithms) to use on the basis of built-in expert knowledge, past cases, meta-learning or using ontological knowledge for planning purposes. Work in the AutoML community [12] has focused on automatically selecting an adequate learning algorithm and setting appropriate hyperparameters for learning a given task. AutoML is based on the Programming by Optimisation 
paradigm [11], which employs algorithm selection and portfolio optimisation. Another fascinating approach is provided by the automated statistician [22], which starts from a data set, automatically fits a statistical model and then generates an explanation of the model in a paper written in natural language.

Characteristic for the existing approaches is that they assume that 1) the learning task is given, and 2) the data is already in the right format for the analysis. However, this is assuming that the most important problem has already been solved. Indeed, since the inception of the field of data mining, people like Usama Fayyad have argued that pre-processing (including the identification of the right targets for predictive modeling) typically takes 80 per cent of the effort in knowledge discovery and the actual data mining step (that is, finding the right model with a system) only requires 20 per cent. The $\mathrm{SYNTH}^{1}$ approach advocated in the present paper aims at supporting all steps of the data analysis problem, including pre-processing and feature selection, identifying the learning task and synthesizing a model for the dataset. As this is clearly a (very) ambitious task, we identify a simple but highly non-trivial setting for studying automated data science in which one is given a set of tables (e.g., worksheets in a spreadsheet) and the task is to automatically complete some of the missing entries. Furthermore, we introduce some elements of an automated data scientist for tackling this task: automated data wrangling, flexible prediction, constraint learning and autocompletion.

\section{Autocompletion in spreadsheets}

Let us introduce the problem studied in this paper using the example provided in Figure 1. It is a typical (very simplified) business example of the use of a spreadsheet. It contains information about the sales of particular flavors of ice-cream in different countries and months, as well as information about the production time taken to produce one unit of ice-cream. Assuming that decisions need to be made about which flavors of ice-cream to retain in which countries, based on the total sales, costs and profitability. However, the left table is incomplete, as the values for August are not yet available, which would be problematic for the decision making process. However, human data analysts could produce reliable estimates for these missing values in order to facilitate the decision making process.

The question tackled in this paper is how to automatically complete such cells under the assumption, of course, that there are underlying regularities in the data and the data has been entered in the tables in a systematic manner.

Solving this problem requires a number of different steps: 1 ) discover the equation $T$ stating that the column Total is equal to the sum of the columns June, July and August; 2) find a predictive model $A$ for the column August using the available data; 3 ) find a predictive model $P$ for the column Profit using the available data; 4) infer the missing values for August using A; 5) infer the missing

\footnotetext{
${ }^{1}$ SYNTH stands for Synthesising Inductive Data Models, and it is the topic of the ERC AdG project no. 694980; cf. https://synth.cs.kuleuven.be/.
} 
values for Total using the equation $T$; and 6 ) infer the missing values for Profit using $P$.

Notice that this autocompletion setting is simple, yet challenging as in general it is not specified which steps need to be taken, that is, the different learning tasks are not given and the only assumption on the data format is that it is in a set of worksheets.

To address these different tasks, our Automic Data Scientist Synth uses a number of different components: SYNTH-A-SizER [25] is an automatic data wrangling system that transforms a dataset into a traditional attribute-value learning format so that standard machine learning systems can be applied (cf. Section 3), Mercs [26] induces versatile predictive models (cf. Section 4), TACLE [15] induces constraints and formula's in spreadsheets (cf. Section 5), and the autocompletion component ties learning and inference together in a probabilistic framework. We now discuss each of these elements in turn.

\begin{tabular}{|c|c|c|c|c|c|c|}
\hline Type & Country & June & July & August & Total & Profit \\
\hline Vanilla & BE & 610 & 190 & 670 & 1470 & YES \\
\hline Banana & BE & 170 & 690 & 520 & 1380 & YES \\
\hline Chocolate & BE & 560 & 320 & 140 & 1020 & YES \\
\hline Banana & DE & 610 & 640 & 320 & 1570 & NO \\
\hline Speculaas & BE & 300 & 270 & 290 & 860 & NO \\
\hline Chocolate & FR & 430 & 350 & & & \\
\hline Banana & DE & 250 & 650 & & & \\
\hline Chocolate & NL & 210 & 280 & & & \\
\hline
\end{tabular}

\begin{tabular}{|c|c|}
\hline Type & ProdTime \\
\hline Chocolate & 60 \\
\hline Banana & 40 \\
\hline Speculaas & 70 \\
\hline Vanilla & 40 \\
\hline
\end{tabular}

Fig. 1: Two tables in a spreadsheet

\section{Data Wrangling}

The focus in the SYNTH setting is on working with spreadsheets while imposing as few assumptions on the user as possible. While it is assumed that the user is systematic, and that there exist regularities in the data that can be exploited by data analysis techniques, it not assumed that the user is able to put the spreadsheet in one of the formats required by standard data analysis and machine learning software. Rather the user should be able to work with the format of the spreadsheet that she created and the required data transformations should be hidden to the user.

That is where automated data wrangling steps in. Automated data wrangling integrates ideas from program synthesis with data science. 
Our SyNTH-A-SizER tackles the following task [25]. Given a dataset $S$ and a machine learning algorithm $M$, find a transformed dataset $D=t(S)$ so that 1) $D$ is in the format required by $M$, and 2) the unknown target model $h$ can be learned (or approximated) by algorithm $M$ on $D$. The transformation $t$ is a program written in a domain specific language and consists of a sequence of simple data transformations. So far, we have made the assumption that the target format is in attribute-value form.

Automated data wrangling has received quite some attention recently. Some data wrangling tools focus on the layout of the data: Trifacta's WRANGLER system provides a graphical interface to transform spreadsheets without writing code and FLASHRELATE [2] allows for data extraction programs to be synthesised from input-output examples. Other tools focus on transforming the data itself to a standardised format $[21,13]$. Common to these approaches is the need for user-guidance, either in the form of examples or of intent. In return, they allow for a large variety of output formats. Within the SYNTH framework, however, it is clear that the transformed data will be used as input to a particular data analysis suite (so far assumed to be in attribute-value format). This imposes strong constraints on the format of transformed data and can be used to minimize the required user interaction. On the other hand, spreadsheets are notorious for being semi-structured. They exhibit some structure as all data is aligned in a grid, but there are no rules on how exactly the structure should be laid out. The same dataset has many representations in spreadsheet format and it is up to the data wrangler to discover and exploit the structure.

To illustrate the SYNTH-A-SIZER setting, consider Figure 2a which contains a small car dealership interested in deciding when to offer discounts. There is also a separate table containing information about the employees pitching the sales. Running any machine learning algorithm on this data requires conversion into an appropriate format, such as attribute-value pairs. The following sequence of transformations (see $[24,25]$ for details of the transformation) yields the desired representation in Figure 2b: Split (Sales, 1), Fill (Sales, 1), Delete (Sales, 2), Join(Sales, Employees, 3, 1). Any attribute-value learning system can be applied on the resulting dataset, e.g., to learn a predictive model $h$ for the last column. This model can then be applied to predict the target value of interest, which in our illustration is indicated by the question mark, and the resulting prediction can be mapped back to the spreadsheet in the original format to hide the details of the transformation and the learning from the end-user. Ideally, the only required input would be for the user to select the question mark. After marking the target value, the system has to figure out an appropriate target representation and synthesize an adequate program.

The main technique employed in our SYNTH-A-SIZER is predictive synthesis, meaning that we predict and evaluate the output of the synthesised program while searching for a solution. The search process is guided by heuristics that are based on two ideas. First, in an attribute-value format, the rows correspond to the examples. Secondly, the attributes or the columns contain values belonging to the same domain. To exploit the first property, we allow the user to mark which 


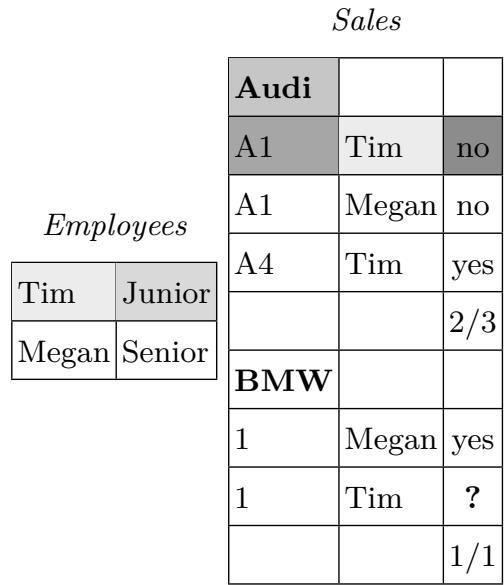

(a) Car dealership data in a raw format

\begin{tabular}{|l|l|l|c|c|}
\hline Tim & Junior & Audi & A1 & no \\
\hline Megan & Senior & Audi & A1 & no \\
\hline Tim & Junior & Audi & A4 & yes \\
\hline Megan & Senior & BMW & 1 & yes \\
\hline Tim & Junior & BMW & 1 & $?$ \\
\hline
\end{tabular}

(b) The data from (a) as a single table and thus in attribute-value format

Fig. 2: Car dealership data.

cells belong together in the worksheets, that is, belong to the same example. The second property also provides a strong constraint on the target transformation, if the domains are given; [24] shows how this constraint can be exploited. In our more recent work [25], however, we heuristically evaluate the degree to which the values in a particular column belong to the same domain. Essentially, the cells in each column are checked for syntactic similarity against a reference cell selected by the user. The syntactic similarity is an edit distance metric on character level, meaning that replacing a digit by another digit is free. The combination of these two properties shows already promising results.

It should be mention that other types transformations could be used as well, for instance, after finding a suitable set of database dependencies, a set of tables could be mapped into a single table using standard database operations such as joins.

We are currently working on reducing the dependency on selecting a full example. By first discovering the domains in the data, syntactically as well as semantically, we can reduce this dependency of the heuristic on the user-input. In addition to changing the layout of the spreadsheets, automated wrangling approaches for data standardisation [21] are also to be incorporated. This step can be facilitated by domain detection [6] and will ultimately be performed without the need for examples - the output format will depend on the domains and on the machine learning algorithm targeted.

\section{Versatile models and Mercs}

Autocompletion of tables requires the use of predictive models that are learned from data. Concerning the learning phase, an important difference with the 
standard setting of supervised learning is that, in the standard setting, the input space and output space are fixed in advance: whether one learns a random forest, a neural network, or any other predictive model, the learning algorithm needs to know which variables are the inputs, and which ones the outputs, before it starts learning.

In the SyNTH setting, the user may not know in advance which variables can most easily be predicted from which other variables. Ideally, a model is learned that in principle is able to predict any variable from any other variables; we call this type of predictive models versatile models. Once such a versatile model is available, it can be analyzed to determine which fields can be predicted from which other fields. This can be used by an intelligent or automatic user interface to reason about autocompletion of the data.

SYNTH will use a recently proposed approach to learning versatile models, called Mercs. Mercs stands for multidirectional ensembles of regression and classification trees. The basic idea behind it is simple. Mercs learns an ensemble of decision trees, where each tree may have a different set of target attributes (as opposed to classical ensembles, where a single target variable is given in advance and all trees try to predict that value); see Figure 3 for an illustration. In its most basic version, Mercs could simply learn a classic ensemble for each variable separately. When there are $k$ trees in an ensemble, and $m$ variables in total, this requires learning $m k$ trees. This number can go down by learning trees that predict multiple variables at the same time, so-called multi-target trees. If each tree in the multidirectional ensemble predicted $v$ variables, then the number of trees required to have each variable predicted by $k$ trees can be divided by $v$. Several authors $[23,19]$ have shown that (ensembles of) multi-target trees often achieve accuracies comparable to that of their single-target counterparts, while being smaller, faster to learn, and faster at prediction time; they achieve state-of-the-art accuracy in a variety of domains.

Another type of models that could be used as versatile predictive models are probabilistic graphical models (PGMs), such as Bayesian networks, Markov networks, etc. The main difference between PGMs and MerCs is that PGMs model a joint probability distribution, whereas MERCs merely model a set of functions. While the latter is implicitly defined by the former, exact probabilistic inference using PGMs is NP-hard, and even approximate inference is NP-hard if guarantees are asked about the quality of the approximation [17].

In the SYNTh context, Mercs is supposed to predict the correct outcome, in those cases where it can be predicted. We do not necessarily want to know the probability of each possible value when there is uncertainty. This is an inherently simpler problem than probabilistic inference, and it may have simpler solutions. That this is indeed the case was shown by Van Wolputte et al. [26], who implemented and evaluated a first version of MERCS. In a comparison with a state-of-the-art Bayesian network learner, MERcs learned models with comparable accuracy and training times, but with inference times that were orders of magnitude faster. In the context of SYNTH, this version of MERCs can be straightforwardly deployed for filling in values in a single table. 


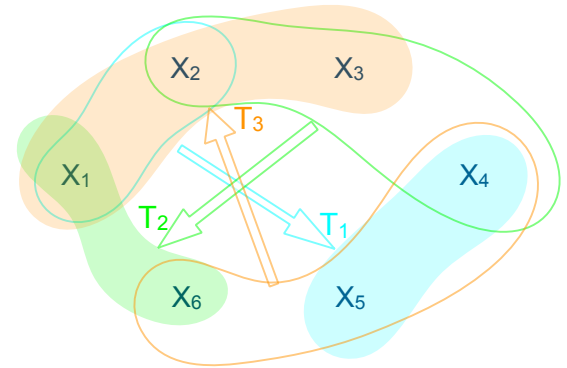

Fig. 3: Schematic illustration of multidirectional ensembles. Each tree $\left(T_{1}, T_{2}\right.$, $T_{3}$ ) takes a different set of input and output variables. In this example, each variable is predicted at least once; $X_{1}$ is predicted by two trees. Having trees simultaneously predict multiple outputs reduces the total number of trees needed to cover each potential target variable with a sufficient number of trees.

Apart from the automatic filling of tables, Mercs can also be used to detect errors. Indeed, any versatile model can be used for detecting anomalies by comparing predicted values with observed values; when both differ, this is an anomaly. Anomalies do not necessary indicate errors (there may be other reasons why a value is anomalous, apart from an entry error), but in many application contexts an entry error may indeed be the most likely cause of an anomaly.

When multiple tables are available, the spreadsheet is essentially a relational database, and predictions in one table may require information from different tables. That is, a relational learner may be required. To this aim, we are currently doing research on a relational version of MERCS, which will use first-order decision trees as learned by the relational learning system TILDE [5].

From a wider machine learning perspective, the MERCs approach leads to a variety of research questions, which are yet to be addressed. For instance:

- The variation in target sets introduces more variation among trees predicting one particular variable. Normally, variation is introduced through training set resampling and, in the case of random forests, randomness in the input attributes considered at each node. In MERCs, the co-targets of a given variable vary among trees, introducing additional variation that may, for instance, render resampling unnecessary.

- A given target variable's value is predicted by combining trees. Some of these trees may test attributes that are missing. In a standard ensemble, those attributes' value must be imputed, or the tree must deal with missing values in some other manner. In a MERCS model, the missing values can be predicted using other trees that have these attributes as targets. Different possible procedures are currently being explored regarding how this can be done. SYNTH's autocompletion setting goes even further in that it wants 
to combine the predictions made by versatile models with inference based on other types of theories, such as TACLE's constraint theories that are discussed next.

\section{Learning Constraints and TacLe}

The goal of TACLE [15] is to discover formulas and constraints in a spreadsheet. It is this component that could induce the equation $T$ in the example for Figure 1. An illustration of TACLE is given in Figures 4 and 5, where the constraints shown in Figure 5 are automatically induced from the three tables in Figure 4.

To this end, TACLE has a set of constraint templates to specify which types of formulas it should look for. Every constraint template is made up of three parts: syntax, signature and definition. An example of a constraint template is column-wise sum, whose syntax is $B_{2}=S U M_{c o l}\left(\mathbf{B}_{1}\right)$, where $B_{2}$ is a column and $\mathbf{B}_{1}$ a set of consecutive columns, i.e., a matrix. By filling in ranges of cell addresses for arguments $\mathbf{B}_{1}$ and $B_{2}$, we obtain an actual constraint that tells us that the $i$-th cell assigned to $B_{2}$ is equal to the sum of the $i$-th column of the cells assigned to $\mathbf{B}_{1}$. Discovering column-wise sum constraints is thus the task of finding assignments of cell ranges to constraint template arguments such that the assignment satisfies the constraint template. Checking whether an assignment satisfies a constraint template is done using the signature and definition of that template. The signature checks necessary conditions on the properties of the ranges, e.g., do they contain the right type of values (numeric for sum) and are the sizes of the ranges compatible, while the definition can be used to look up the actual values of the cells in those ranges, to compute their sums and to verify whether the results match.

The main challenges for learning constraints are dealing with the vast amount of possible assignments to every constraint template and avoiding the discovery of spurious constraints. To deal with these problems, TACLE includes a preprocessing step to convert spreadsheets to a more structured representation and subsequently tries to prune impossible sets of assignments from its search.

Internally, TACLE reasons over tables of equally-sized rows and/or columns, blocks which are continuous ranges of rows or columns with the same type (e.g., numeric or textual) and finally individual vectors (a row or a column) of typeconsistent cells. Tables in a spreadsheet are detected either automatically or using a visual selection tool and can be automatically split into a minimal number of type-consistent blocks. Blocks group together neighboring vectors of the same type and vectors form the minimal level of granularity, i.e., constraints must always reason about entire vectors. For this initial step, there is clearly room for cross-fertilization between TACLE's pre-processing steps and SYNTH-A-SizER's automatic transformation process.

TACLE employs a couple of strategies to prune impossible assignments.

First, when finding valid assignments for a constraint template, it considers two levels of granularity: reasoning over entire type-consistent blocks as detected in the previous phase (input blocks) and then reasoning over assignments of sub- 
sets of input blocks (subblocks and vectors). After precomputing the properties of every input block, it uses a constraint satisfation solver to find assignments of input blocks to constraint template arguments that are compatible with the constraint template signature. Compatibility means that subsets of the input blocks could potentially satisfy the signature. This step eliminates whole sets of assignments that are incompatible. For every valid input block assignment TACLE generates all assignments of subblocks and tests if they fulfil the signature and definition.

Secondly, TACLE also considers dependencies between constraint templates. If a constraint template $s_{2}$ requires a constraint of type $s_{1}$ to hold on a subset $A_{d}$ of its arguments $A$, then the search for valid assignments to $s_{2}$ is bootstrapped. Instead of generating all possible assignments to $A$, the values for $A_{d}$ are prepopulated with valid assignments to $s_{2}$.

\begin{tabular}{|c|c|c|c|c|c|c|c|c|c|}
\hline ID & Salesperson & Q1 & Q2 & Q3 & Q4 & Total & Rank & & \\
\hline 1 & James Smith & 353 & 378 & 396 & 387 & 1514 & 2 & & \\
\hline 2 & Maria Garcia & 370 & 408 & 387 & 386 & 1551 & 1 & & \\
\hline 3 & Micheal Jones & 175 & 146 & 167 & 203 & 691 & 3 & & \\
\hline 4 & Grace Hartman & 93 & 98 & 96 & 105 & 392 & 4 & & \\
\hline Block 1 & Block 2 & \multicolumn{6}{|c|}{ Block 3} & & \\
\hline$T_{1}[:, 1]$ & $T_{1}[:, 2]$ & \multicolumn{6}{|c|}{$T_{1}[:, 3: 8]$} & & \\
\hline & & & & & & & Customer & Contact & Contact Name \\
\hline Total & 991 & 1030 & 1046 & 1081 & 4148 & & Frank & 1 & James Smith \\
\hline Average & 247.75 & 257.5 & 261.5 & 270.25 & 1037 & & Sarah & 3 & Micheal Jones \\
\hline Max & 370 & 408 & 396 & 387 & 1551 & & George & 3 & Micheal Jones \\
\hline \multirow[t]{4}{*}{ Min } & 93 & 98 & 96 & 105 & 392 & & Mary & 2 & Maria Garcia \\
\hline & \multicolumn{5}{|c|}{ Block 4} & & Tim & 4 & Grace Hartman \\
\hline & \multicolumn{5}{|c|}{$T_{2}[1: 4,:]$} & & Block 5 & Block 6 & Block 7 \\
\hline & & & & & & & $T_{3}[:, 1]$ & $T_{3}[:, 2]$ & $T_{3}[:, 3]$ \\
\hline
\end{tabular}

Fig. 4: Example spreadsheet. For illustration purposes (not present in the spreadsheet), gray coloring is used to show detected blocks (light gray for blocks with numeric data and darker gray for blocks with textual data) and block names and notations are provided in italic.

TACLE is one example of a system that learn constraints from examples. While constraints are ubiquitous in artificial intelligence, the learning of constraint theories has not received a lot of attention, but see [8] for an overview of the state-of-the-art and $[4,3]$ for particular approaches coming from the field of constraint programming. TACLE and SYNTH-A-SizER are also inspired on the program synthesis line of work originated in FLASHFILL [10]. 
one or more spreadsheets. The autocompletion loop involves both a user, who is filling out one or more spreadsheets, and the SYNTH system, which continuously suggests potential values to be filled into the cells, rows, or columns that the user is currently working on. The suggestions are presented in a sidebar, so that the user can accept or reject them without being interrupted while working on the spreadsheet. The user can also select a range of cells and request to autocomplete them. Whenever the user updates the values of any cells or rejects a proposed values, the SYNTH system updates the relevant predictors and re-evaluates its confidence. The idea is that in this way SYNTH learns from the user feedback.

\section{Conclusions}

We have defined the SYNTH challenge for automated data science and described some elements of an initial attempts to tackle it. Essential for solving the autocompletion task is the ability to learn constraints and versatile predictive models, ways to deal with the data wrangling aspects, as well as techniques for deciding what to learn and how to perform inference with the resulting models.

\section{Acknowledgements}

This work has received funding from the European Research Council (ERC) under the European Union's Horizon 2020 research and innovation programme (grant agreement No [694980] Synth: Synthesising Inductive Data Models) and the Research Foundation, Flanders.

\section{References}

1. Bot.me: How artificial intelligence is pushing man and machine closer together. Tech. rep., PwC (2017)

2. Barowy, D.W., Gulwani, S., Hart, T., Zorn, B.: Flashrelate: Extracting relational data from semi-structured spreadsheets using examples. SIGPLAN Not. 50(6), 218-228 (Jun 2015)

3. Beldiceanu, N., Simonis, H.: A model seeker: Extracting global constraint models from positive examples. In: Proceedings 18th International Conference on Principles and Practice of Constraint Programming. Lecture Notes in Computer Science, vol. 7514, pp. 141-157 (2012)

4. Bessiere, C., Koriche, F., Lazaar, N., O'Sullivan, B.: Constraint acquisition. Artif. Intell. 244, 315-342 (2017)

5. Blockeel, H., De Raedt, L.: Top-down induction of first-order logical decision trees. Artif. Intell. 101(1-2), 285-297 (1998)

6. Contreras-Ochando, L., Martínez-Plumed, F., Ferri, C., Hernández-Orallo, J., Ramírez-Quintana, M.J., Katayama, S.: Domain specific induction for data wrangling automation (demo). AutoML @ ICML 2017 (2017)

7. De Raedt, L., Kimmig, A., Toivonen, H.: Problog: A probabilistic prolog and its application in link discovery. In: Proceedings 20th International Joint Conference on Artificial Intelligence (2007) 
8. De Raedt, L., Passerini, A., Teso, S.: Learning constraints from examples. In: Proceedings 32nd AAAI Conference on Artificial Intelligence (2018)

9. Fierens, D., Van den Broeck, G., Renkens, J., Shterionov, D., Gutmann, B., Thon, I., Janssens, G., De Raedt, L.: Inference and learning in probabilistic logic programs using weighted boolean formulas. Theory and Practice of Logic Programming 15(3), 358-401 (2015)

10. Gulwani, S.: Automating string processing in spreadsheets using input-output examples. In: ACM SIGPLAN-SIGACT. pp. 317-330. POPL (2011)

11. Hoos, H.H.: Programming by optimization. Communications of the ACM 55(2), 70-80 (2012)

12. Hutter, F., Kotthoff, L., Vanschoren, J. (eds.): AutoML: Methods, Systems, Challenges (2018), draft available from https://www.ml4aad.org/book/

13. Jin, Z., Cafarella, M., Jagadish, H., Kandel, S., Minar, M.: Unifacta: Profilingdriven string pattern standardization. arXiv preprint arXiv:1803.00701 (2018)

14. King, R.D., Whelan, K.E., Jones, F.M., Reiser, P., Bryant, C.H., Muggleton, S., Kell, D., Oliver, S.: Functional genomic hypothesis generation and experimentation by a robot scientist. Nature 427, 247-252 (2004)

15. Kolb, S., Paramonov, S., Guns, T., De Raedt, L.: Learning constraints in spreadsheets and tabular data. Machine Learning 106(9-10), 1441-1468 (2017)

16. Kurzweil, R.: The age of intelligent machines. MIT press Cambridge, MA (1990)

17. Kwisthout, J.: Approximate inference in bayesian networks: Parameterized complexity results. Int. J. Approx. Reasoning 93, 119-131 (2018)

18. Russell, S., Norvig, P.: Artificial Intelligence: a Modern Approach. Prentice Hall, 3rd edn. (2010)

19. Schietgat, L., Vens, C., Struyf, J., Blockeel, H., Kocev, D., Dzeroski, S.: Predicting gene function using hierarchical multi-label decision tree ensembles. BMC Bioinformatics 11(2) (2010)

20. Serban, F., Vanschoren, J., Kietz, J.U., Bernstein, A.: A survey of intelligent assistants for data analysis. ACM Computing Surveys (CSUR) 45(3) (2013)

21. Singh, R., Gulwani, S.: Transforming spreadsheet data types using examples. Sigplan Notices 51(1), 343-356 (2016)

22. Steinruecken, C., Smith, E., Janz, D., Lloyd, J., Ghahramani, Z.: The automated statistician (2018), draft available from https://www.ml4aad.org/book/

23. Vens, C., Struyf, J., Schietgat, L., Dzeroski, S., Blockeel, H.: Decision trees for hierarchical multi-label classification. Machine Learning 73(2), 185-214 (2008)

24. Verbruggen, G., De Raedt, L.: Towards automated relational data wrangling. In: Proceedings of AutoML 2017@ ECML-PKDD: Automatic selection, configuration and composition of machine learning algorithms. pp. 18-26 (2017)

25. Verbruggen, G., De Raedt, L.: Automatically wrangling spreadsheets into machine learning data formats. In: Proceedings of the Intelligent Data Analysis Symposium. Lecture Notes in Computer Science, vol. this volume. Springer Verlag (2018)

26. Wolputte, E.V., Korneva, E., Blockeel, H.: MERCS: multi-directional ensembles of regression and classification trees. In: Proceedings 32nd AAAI Conference on Artificial Intelligence (2018) 